УДК 614.8; 621.315

ПОВЫШЕНИЕ ЭФФЕКТИВНОСТИ ЭКСПЛУАТАЦИИ

КАБЕЛЬНЫХ ЛИНИЙ С ИСПОЛЬЗОВАНИЕМ

КОМБИНИРОВАННОЙ ДИАГНОСТИКИ

\title{
IMPROVING THE EFFICIENCY OF OPERATING CABLE LINES USING COMBINED DIAGNOSTICS
}

\author{
Ф.Ш. Хафизов, Ж. да К. Новикова Фрейре Шавиер
}

Уфимский государственный нефтяной технический университет, г. Уфа, Российская Федерация

Fanil Sh. Khafizov, Gessiane da Konseysau Novikova Freyre Shavier

Ufa State Petroleum Technological University, Ufa, Russian Federation

e-mail: pkpb@mail.ru

Аннотация. Значение кабельных линий в системах электроснабжения предприятий резко возрастает в связи с развитием промышленности, так как они являются важными, ответственными и дорогостоящими элементами систем электроснабжения. В связи с этим требуется большой срок эксплуатации кабельных линий, что обуславливает потребность разработки и внедрения мероприятий по повышению их эффективности и надёжности. Надёжность их работы - это одна из важнейших задач бесперебойного электроснабжения для предприятий, которые относятся к первой и второй категории потребителей, где непрерывность технологии производства очень важна. Резкая остановка производства связана с высокими потерями и рисками как экономического, так и техногенного характера.

В настоящее время, согласно данным Открытого акционерного общества «Федеральная сетевая компания Единой энергетической 
системы» (ОАО «ФСК ЕЭС»), кабельные линии морально и физически изношены на 50-70 \%, что является причиной их частых аварийных отказов.

Повышение эффективности и уровня эксплуатационной надежности кабельных линий можно достигнуть, используя комбинированный метод диагностики, учитывая все технические параметры кабелей. На основе экспериментальных данных, полученных при помощи комбинированной диагностики, была установлена взаимосвязь диагностических параметров с характерными повреждениями кабельных линий. Анализ результатов исследований позволил построить диаграмму «паутина», по которой делается вывод о техническом состоянии кабельной линии. На основе данной диаграммы получена количественная оценка их уровня поврежденности, которая позволяет получить повышение эффективности эксплуатации кабельных линий.

Abstract. The importance of cable lines in enterprises power supply systems increases due to the industry development, as they are important, responsible and expensive elements of power supply systems. In this connection, a long service life of cable lines is required, which necessitates the development and implementation of measures to improve their efficiency and reliability. The their work reliability is one of the most important tasks of uninterrupted power supply for enterprises that belong to the first and second category of consumers, where production technology is very important. An abrupt halt in production is associated with high losses and risks, both economic and man-made.

At present, according to the data of the Joint Stock Company Federal Grid Company of the Unified Energy System (JSC FGC UES), cable lines are morally and physically worn out by 50-70\%, which is the reason for their frequent crashes.

Improving the efficiency and operational reliability level of cable lines can be achieved using a combined diagnostic method, taking into account all the technical parameters of cables. On the experimental data basis obtained using 
the combined diagnostics, the diagnostic parameters relationship with the characteristic damage to cable lines was established. Analysis of the research results allowed to construct a «web» diagram, which makes a conclusion about the technical condition of the cable line. Based on this diagram, a quantitative assessment of their damage level was obtained, which allows to obtain an increase in the cable lines operation efficiency.

Ключевые слова: эффективность, аварийные отказы, кабельные линии, надёжность, диагностика, технические параметры, неразрушающий контроль

Key words: efficiency, emergency failures, cable lines, reliability, diagnostics, technical parameters, nondestructive check

Значение кабельных линий в системах электроснабжения предприятий резко возрастает в связи с развитием промышленности, так как они являются важными, ответственными и дорогостоящими элементами систем электроснабжения. Надёжность работы кабельных линий - это одна из важнейших задач бесперебойного электроснабжения для предприятий, которые относятся к первой и второй категории потребителей, где непрерывность технологии производства очень важна. Резкая остановка производства связана с высокими потерями и рисками как экономического, так и техногенного характера.

Одной из главных причин аварийных отказов и простоев на предприятиях является моральный, а также физический износ кабельных линий. Согласно данным ОАО «ФСК ЕЭС» кабельные линии морально и физически изношены на 50-70 \%. Анализ статистических данных с 2003 г. о пожарах, согласно ВНИИПО МЧС РФ, показывает, что самыми пожароопасными являются кабели и кабельные изделия [1]. В таблице 1 приведены статистические данные по пожарам, связанным с кабельными изделиями. 
Таблица 1. Статистические данные по пожарам от кабельных изделий

\begin{tabular}{|c|c|c|c|}
\hline \multicolumn{4}{|c|}{ Кабельные изделия (кабель, провод) } \\
\hline Пожары, \% & Прямой ущерб, \% & Травмы, \% & Погибло, \% \\
\hline 61,5 & 55 & 44,3 & 32 \\
\hline
\end{tabular}

Проанализировав статистические данные об аварийных отказах и о пожарах, можно сделать вывод о том, что одной из важных задач, согласно принятой «Концепции обеспечения надежности в электроэнергетике», является повышение эффективности и уровня эксплуатационной надежности кабельных линий. Это можно достигнуть благодаря использованию комбинированного метода диагностики, изучая и учитывая все технические параметры кабелей [2].

Использование комбинированной диагностики подразумевает сочетание уже известных, но модернизированных и только неразрушающих методов диагностики кабельных линий. Все современные методы оценки технического состояния приведены на рисунке 1.

Сочетание неразрушающих методов позволит объединить сбор данных, их анализ, выявление проблемных мест и прогнозирование остаточного ресурса кабельной линии [3].

Данный подход актуален как для повышения эффективности эксплуатации кабельных линий, так и для увеличения их надёжности. Комбинированный метод диагностики также должен учитывать все требования нормативных документов, таких как ПУЭ (правила устройства электроустановок), ГОСТ 12.2.007.14-75, РД 34.45-51.300-97 и другие [4].

Для реализации комбинированной диагностики была создана лабораторная установка, представленная на рисунке 2. 


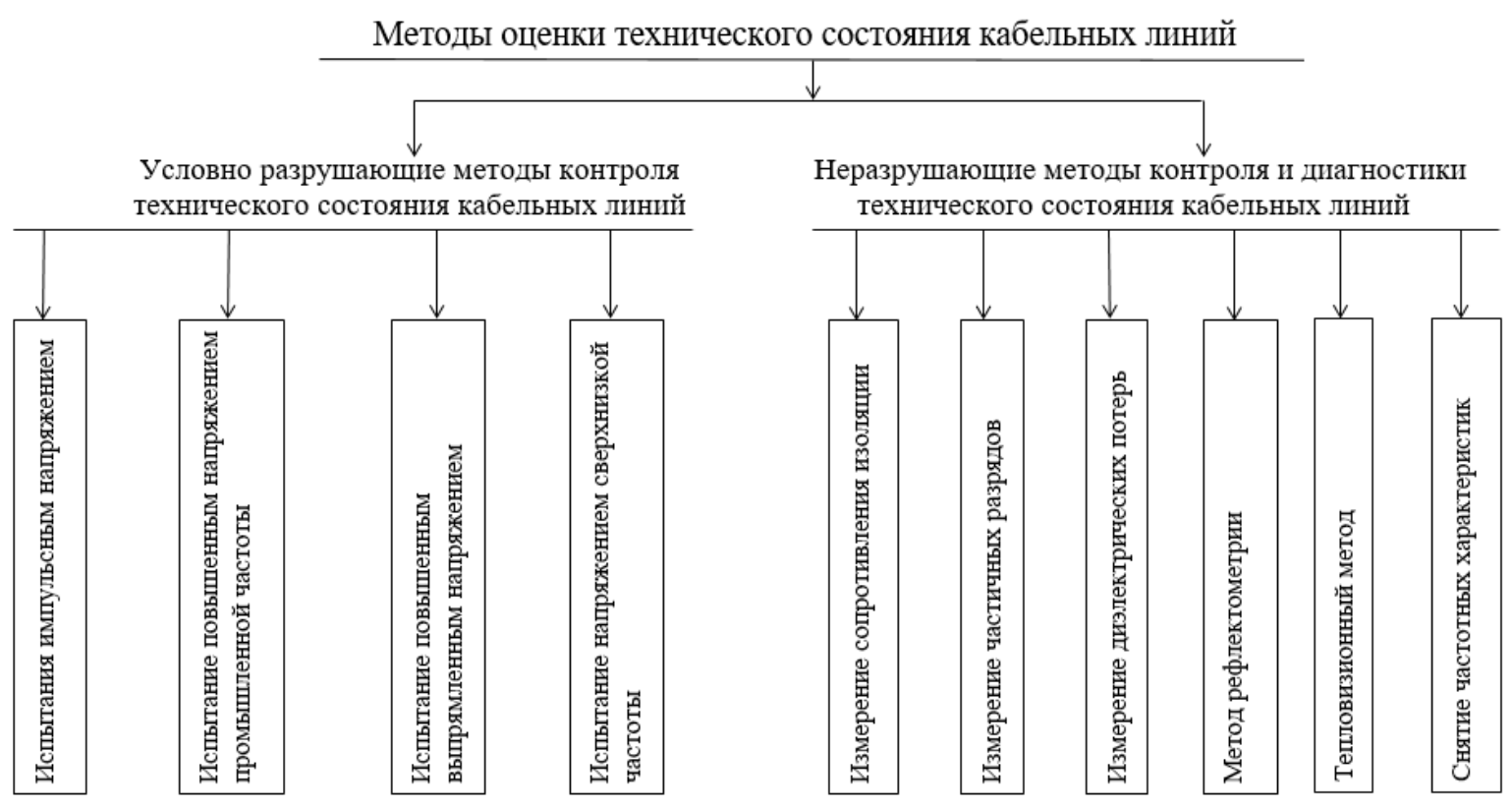

Рисунок 1. Современные методы оценки кабельных линий

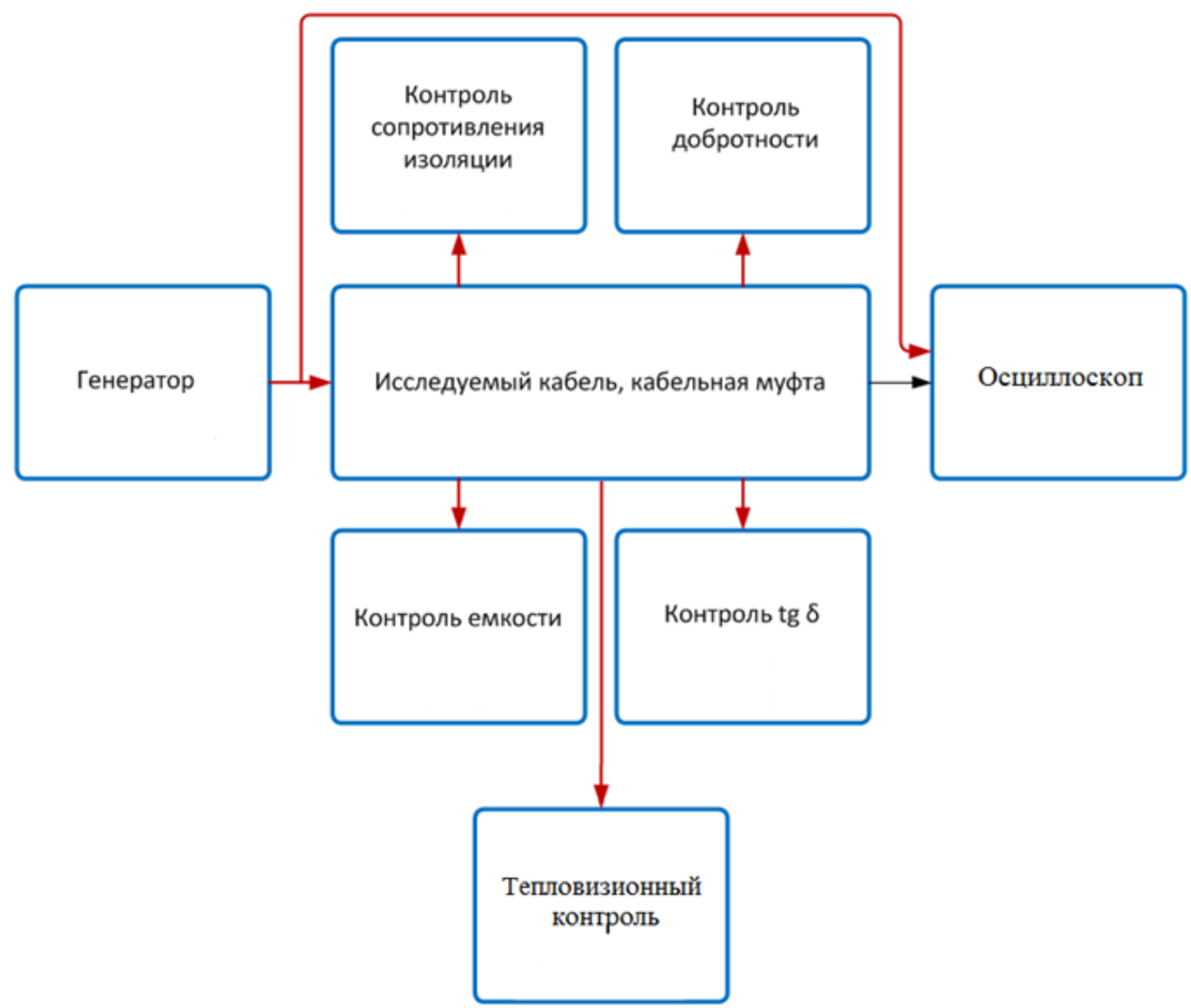

Рисунок 2. Схема лабораторной установки

Проводились измерения таких параметров кабельных линий, как:

- сопротивление изоляции;

- добротность; 
- емкость;

- тангенс диэлектрических потерь.

Для более точного контроля состояния кабельной линии использовали метод неразрушающего контроля - тепловизионный контроль [5].

Полученные результаты измерений внесли в таблицу 2, заранее условно разделив кабели на три технических состояния: исправный, увлажненный и деформированный.

Таблица 2. Результаты измерений параметров кабелей

\begin{tabular}{|c|c|c|c|c|c|c|c|}
\hline \multicolumn{2}{|c|}{ Фаза } & A & B & $\mathrm{C}$ & $\mathrm{AB}$ & $\mathrm{BC}$ & $\mathrm{AC}$ \\
\hline \multirow{5}{*}{ Исправный } & Емкость С, нФ & 0,449 & 0,454 & 0,458 & 0,271 & 0,271 & 0,274 \\
\hline & $\begin{array}{l}\text { Тангенс угла } \\
\text { диэлектрических } \\
\text { потерь } \operatorname{tg} \delta\end{array}$ & 0,002 & 0,002 & 0,002 & 0,024 & 0,022 & 0,024 \\
\hline & Добротность Q & 72,10 & 69,21 & 69,21 & 70,00 & 70,25 & 67,71 \\
\hline & $\begin{array}{l}\text { Сопротивление } \\
\text { изоляции R, MOм }\end{array}$ & \multicolumn{6}{|c|}{100} \\
\hline & $\begin{array}{l}\text { Избыточная } \\
\text { температура, }{ }^{\circ} \mathrm{C}\end{array}$ & \multicolumn{6}{|c|}{5} \\
\hline \multirow{5}{*}{ Увлажненный } & Емкость С, нФ & 0,871 & 0,451 & 0,454 & 0,422 & 0,270 & 0,424 \\
\hline & $\begin{array}{l}\text { Тангенс угла } \\
\text { диэлектрических } \\
\text { потерь tg } \delta\end{array}$ & 0,309 & 0,002 & 0,002 & 0,082 & 0,003 & 0,082 \\
\hline & Добротность Q & 66,92 & 67,52 & 66,87 & 12,7 & 317,4 & 15,32 \\
\hline & $\begin{array}{l}\text { Сопротивление } \\
\text { изоляции } \mathrm{R}, \mathrm{MOM}\end{array}$ & \multicolumn{6}{|c|}{36} \\
\hline & $\begin{array}{l}\text { Избыточная } \\
\text { температура, }{ }^{\circ} \mathrm{C}\end{array}$ & \multicolumn{6}{|c|}{25} \\
\hline \multirow{5}{*}{ Деформированный } & Емкость С, нФ & 1,185 & 0,455 & 0,466 & 0,355 & 0,274 & 0,360 \\
\hline & $\begin{array}{l}\text { Тангенс угла } \\
\text { диэлектрических } \\
\text { потерь tg } \delta\end{array}$ & 0,796 & 0,004 & 0,004 & 0,148 & 0,003 & 0,151 \\
\hline & Добротность Q & 1,77 & 261,00 & 96,00 & 10,11 & 207,00 & 13,50 \\
\hline & $\begin{array}{l}\text { Сопротивление } \\
\text { изоляции R, MOм }\end{array}$ & \multicolumn{6}{|c|}{0,5} \\
\hline & $\begin{array}{l}\text { Избыточная } \\
\text { температура, }{ }^{\circ} \mathrm{C}\end{array}$ & \multicolumn{6}{|c|}{43} \\
\hline
\end{tabular}


В результате анализа результатов измерений параметров состояния кабелей, была выделено три группы:

- «нормальное» равно состоянию исправного кабеля;

- «удовлетворительное» равно состоянию кабеля, при котором развивается дефект;

- «неудовлетворительное» равно аварийному состоянию кабеля.

Далее полученные результаты были внесены в диаграмму, где значение каждого параметра откладывается вдоль каждой оси, т.е. построена диаграмма «паутина» [6]. Одна из таких диаграмм представлена на рисунке 3.

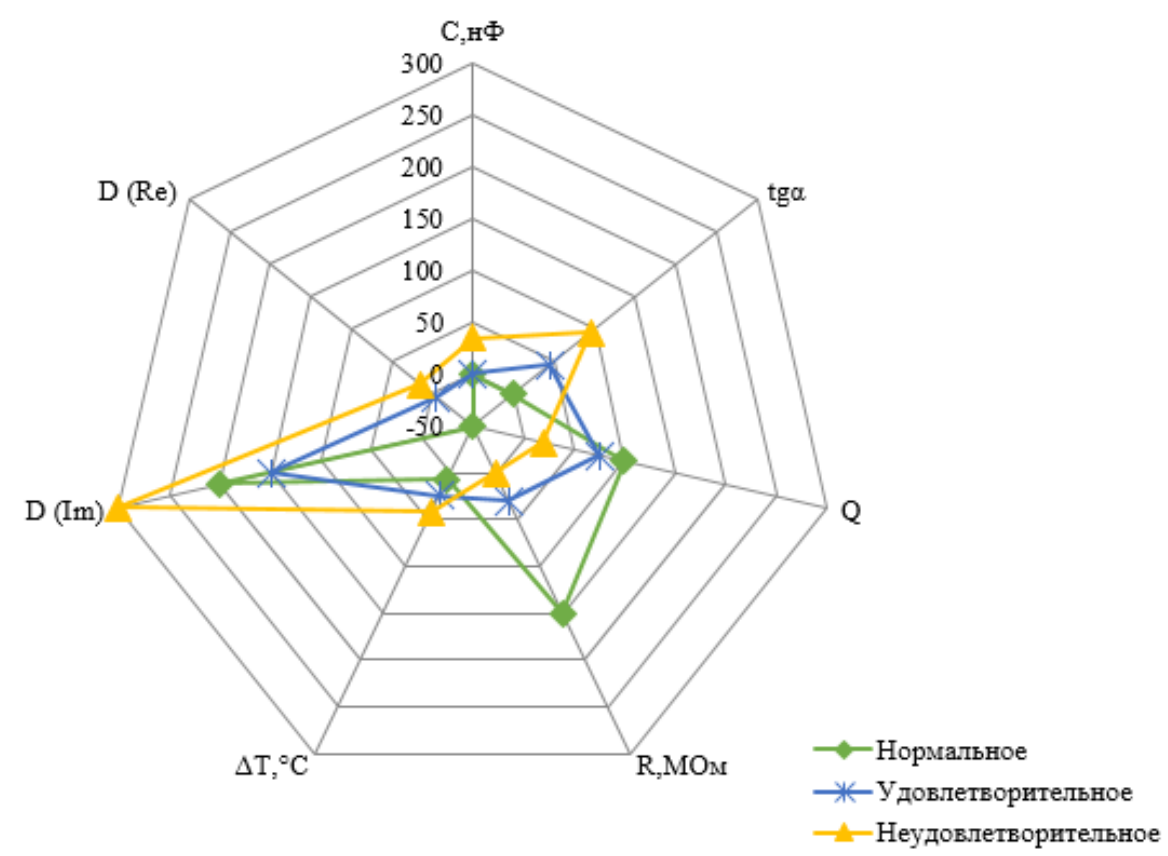

Рисунок 3. Диаграмма «паутина» по оценке состояния кабельных линий

По построенной «паутине» можно рассчитать площадь полученной фигуры и сделать вывод, какое состояние имеет кабельная линия («нормальное», «удовлетворительное», «неудовлетворительное»).

\section{Выводы}

1. На основе экспериментальных данных, полученных при помощи комбинированной диагностики, была установлена взаимосвязь 
диагностических параметров с характерными повреждениями кабельных линий.

2. Анализ результатов исследований позволил построить диаграмму «паутина», на основании которой можно сделать вывод о техническом состоянии кабельной линии. С целью повышения эффективности эксплуатации кабельных линий на основе диаграммы получена количественная оценка уровня их поврежденности.

\section{Список используемых источников}

1. ГОСТ 12.1.033-81. Система стандартов безопасности труда (ССБТ). Пожарная безопасность. Термины и определения. URL: http://docs.cntd.ru/document/1200003841 (дата обращения: 07.01.2019).

2. ГОСТ 22220-76. Кабели, провода и шнуры. Методы определения стойкости изоляции и оболочек из поливинилхлоридного пластика к растрескиванию и деформации при повышенной температуре. URL: http://docs.cntd.ru/document/1200012307 (дата обращения: 07.01.2019).

3. Altug S. Fuzzy Inference Systems Implemented on Neural Architectures for Motor Fault Detection and Diagnosis // IEEE Transactions on Industrial Electronics. 1999. Vol. 46. No. 6. P. 1069-1079.

4. Новикова Фрейре Шавиер Ж. да К., Хафизов Ф.Ш., Хафизов И.Ф. Применение интегральных критериев с целью обеспечения промышленной безопасности при эксплуатации кабельных линий // Сетевое издание «Нефтегазовое дело». 2018. № 6. С. 128-142. URL: http://ogbus.ru/files/ogbus/issues/6_2018/ogbus_6_2018_p128-142.pdf (дата обращения: 07.01.2019).

5. Dugan R.C., McGranaghan M.F., Beaty H.W. Electrical Power Systems Quality. McGraw-Hill, 1996. 265 p.

6. Дементьев В.С. Определение места повреждения силовых кабельных линий. М.: Энергоатомиздат, 1962.96 с. 


\section{References}

1. GOST 12.1.033-81. Sistema standartov bezopasnosti truda (SSBT). Pozharnaya bezopasnost'. Terminy i opredeleniya [State Standard 12.1.033-81. Occupational Safety Standards System (PRS). Fire Safety. Terms and Definitions]. Available at: http://docs.cntd.ru/document/1200003841 (accessed 07.01.2019). [in Russian].

2. GOST 22220-76. Kabeli, provoda i shnury. Metody opredeleniya stojkosti izolyacii $i$ obolochek $i z$ polivinilhloridnogo plastika $k$ rastreskivaniyu $i$ deformacii pri povyshennoj temperature [State Standard 22220-76. Cables, Wires and Cords. Methods for Determining the Resistance of Insulation and Shells of Polyvinylchloride Plastic to Cracking and Deformation at Elevated Temperature]. Available at: http://docs.cntd.ru/document/1200012307 (accessed 07.01.2019). [in Russian].

3. Altug S. Fuzzy Inference Systems Implemented on Neural Architectures for Motor Fault Detection and Diagnosis. IEEE Transactions on Industrial Electronics, 1999, Vol. 46, No. 6.

4. Novikova Freire Shavier Zh. da K., Khafizov F.Sh., Khafizov I.F. Primenenie integral'nyh kriteriev s cel'yu obespecheniya promyshlennoj bezopasnosti pri ehkspluatacii kabel'nyh linij [Integrated Criteria Application to Ensure Industrial Safety Operation of Cable Lines]. Setevoe izdanie «Neftegazovoe delo»-Online Edition «Oil and Gas Business», 2018, No. 6, pp. 128-142. URL: http://ogbus.ru/files/ogbus/issues/6_2018/ogbus_6_2018_p128142.pdf [in Russian].

5. Dugan R.C., McGranaghan M.F., Beaty H.W. Electrical Power Systems Quality. McGraw-Hill, 1996. 265 p.

6. Dementiev V.S. Opredelenie mesta povrezhdeniya silovykh kabel'nykh liniy [Determination of the Place of Damage of Power Cable Lines]. Moscow, Energoatomizdat, 1962.96 p. [in Russian]. 


\section{Сведения об авторах}

\section{About the authors}

Хафизов Фаниль Шамильевич, д-р техн. наук, профессор, заведующий кафедрой «Пожарная и промышленная безопасность», УГНТУ, г. Уфа, Российская Федерация

Fanil Sh. Khafizov, Doctor of Engineering Sciences, Professor, Head of Fire and Industrial Safety Department, USPTU, Ufa, Russian Federation

e-mail:pkpb@mail.ru

Новикова Фрейре Шавиер Жессиане да Консейсау, аспирант кафедры «Пожарная и промышленная безопасность», УГНТУ, г. Уфа, Российская Федерация

Gessiane da Konseysau Novikova Freyre Shavier, Post-graduate Student of Fire and Industrial Safety Department, USPTU, Ufa, Russian Federation e-mail: shavier_93@mail.ru 